\title{
Spherical goniometry and spacecraft attitude determination
}

\author{
Luc Fraiture and Karlheinz Spindler
}

\begin{abstract}
Luc Fraiture received his Ph.D. in mathematical physics at the University of Louvain (Leuven) in Belgium in 1964. In the year 1968 he joined the predecessor organisation of today's European Space Agency (ESA), where he was in charge of satellite control for 28 years. Having retired from ESA, he now dedicates much of his free time to performing studies related to spacecraft attitude determination and control.

Karlheinz Spindler received his mathematical education at the Technische Hochschule Darmstadt. After receiving his Ph.D. in mathematics, he spent two years as a visiting assistant professor at Louisiana State University in Baton Rouge (USA) and then worked for five years in the Flight Dynamics Department of the European Space Operations Centre (ESOC) in Darmstadt. He currently teaches mathematics and data processing at the Fachhochschule Wiesbaden. His research interests include geometric methods in control theory and parameter estimation methods related to the study of dynamical systems.
\end{abstract}

\section{Introduction}

Goniometry, literally the science of measuring angles, is defined in [8] as "the part of trigonometry determined by trigonometric functions and their relations" (vol. 4, p. 287),

Bei der Lagebestimmung von Satelliten geht es darum, die räumliche Orientierung eines Satelliten aus Sensordaten zu ermitteln, bei denen es sich in der Regel um Richtungsmessungen handelt. In der gängigen Praxis werden diese Richtungen in Form von Vektorkoordinaten codiert, was eine gewisse Redundanz an Information mit sich bringt, insbesondere dann, wenn nicht absolute Richtungen, sondern nur relative Unterschiede zwischen verschiedenen Richtungen von Bedeutung sind. Wie in dem vorliegenden Artikel gezeigt wird, lassen sich manche Lagebestimmungsalgorithmen enorm verbessern, indem statt der Benutzung von Vektoren eine Beschreibung der auftretenden Konstellationen von Richtungen durch gewisse Winkelgrößen gewählt wird. Interessanterweise wird aber die hier beschriebene Vorgehensweise erst durch die Verallgemeinerung auf Sphären beliebiger Dimension wirklich klar. Die Anwendung dieser allgemeinen Ergebnisse auf das Ausgangsproblem der Lagebestimmung von Satelliten erweist dann deren praktische Nützlichkeit. 
encompassing plane trigonometry and spherical trigonometry. From a broader point of view, goniometry can be seen as the study of relations between various angles in geometric configurations on arbitrary Riemannian manifolds. The term is also used in application areas such as orthopedics (see [14]) and crystallography (see [3], [15]). In this paper we apply methods from goniometry to a problem in spacecraft attitude determination, which is the discipline dealing with the task of exploiting directional information derived from sensors to determine the orientation of a satellite in space.

In this discipline it has become customary to indiscriminately code all available directional information in terms of vector coordinates which are then processed in the attitude determination software; spherical trigonometry, to the detriment of the solution, is often insufficiently considered. (This seems to reflect the nearly complete elimination of spherical trigonometry from typical curricula in which it appears, if at all, as an application of vector algebra.) It struck us that this way a dead weight of redundant information is carried through the calculations which is not really needed and often even blurs the real problem. In particular, vector coordinates always carry absolute information whereas quite often only relative information on the mutual relations between different directions is relevant. Treating directions not as vectors, but as objects in their own right (which can be simply thought of as points on the unit sphere) will allow us to deal with sets of directions modulo orthogonal transformations and hence process only relevant information.

While all that is needed to satisfactorily treat attitude determination problems is a judicious use of spherical trigonometry, we found it beneficial in the course of our work to extend spherical trigonometry to arbitrary finite dimensions. (Such an approach is quite natural from the point of view of classical geometry; see Chapter 18, Section 6 in [1] and Book 2, Chapter 5 in [4].) Thus we develop a theory of spherical trigonometry in an arbitrary (real or complex) vector space of finite dimension which is equipped with an inner product (which allows us to compute lengths and angles) and with a determinant function (which allows us to distinguish between different orientations). Our driving theme is the translation of statements about geometric directions from the language of vectors into the language of angles and vice versa. The generalization to arbitrary dimensions provides the conceptual clarity which not only helped us to find an effective solution to the specific problem at hand, but also extends the field of potential applications; we feel that our approach may be useful to tackle problems in other areas (such as pattern recognition) which can be cast as problems of identifying constellations of direction vectors. Moreover, should we wake up one morning and find out that the world's dimension has changed over night, we shall find ourselves all the better prepared (cf. [16], p. 393).

\section{Attitude determination using GPS data}

The Global Positioning System (GPS) is a constellation of currently 29 earth-orbiting satellites (with a minimum of 24 in regular operation) transmitting signals which allow users to perform highly accurate position measurements on earth, for example for navigation and surveying tasks. GPS measurements can also be used to determine the orbital position of a spacecraft (called host spacecraft) receiving signals from GPS satellites. Moreover, GPS measurements have been increasingly used to determine the spatial orientation (attitude) of the host spacecraft (see [2] and [12]), and this is the problem we 
want to study in this paper. We only deal with static (instantaneous) attitude determination and do not make use of kinematical relations which lead to more reliable but initially delayed results. The host spacecraft, whose attitude is to be determined, carries two or more receiving antennas all handling the same GPS signals; the antenna baselines are defined to be the direction vectors between any two of these antennas. The angle of incidence $\beta_{i j}$ from the $i$-th GPS satellite with respect to the $j$-th antenna baseline can be computed from the phase difference of the plane wave from the GPS satellite with respect to the two antennas. This phase difference, however, is only known up to a multiple of the wavelength, and this ambiguity needs to be resolved for each separate GPS satellite signal received by each separate baseline. In fact, as depicted in Fig. 1, we have for each GPS satellite in the direction $v_{i}$ and each separate baseline direction $u_{j}$ with known baseline length $U_{j}$ a measurement $\Phi_{i j}$ and an unknown trigonometric angle $\beta_{i j}$ given by the formula

$$
U_{j} \cos \beta_{i j}=\Phi_{i j}+m_{i j} \lambda
$$

where the known wavelength of the GPS signals is denoted by $\lambda$ and where $m_{i j}$ is an unknown number of wavelength cycles. The wavelength $\lambda$ is either $19 \mathrm{~cm}$ or $24 \mathrm{~cm}$ (according to which of the two GPS transmission frequencies is received); typical values for the baseline lengths $U_{j}$ are in the range between $1 \mathrm{~m}$ and $2 \mathrm{~m}$. We shall denote by $k$ the number of GPS directions and by $\ell$ the number of antenna baselines.

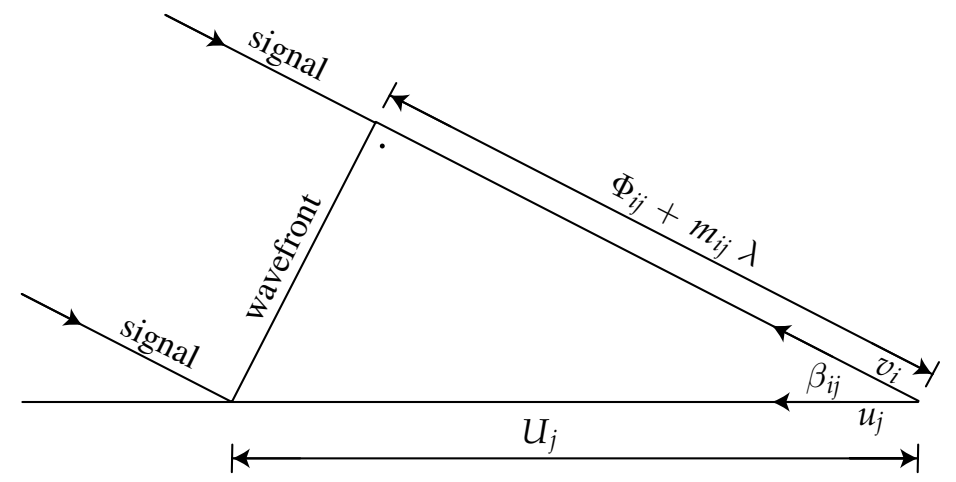

Fig. 1 Reception of a GPS signal by two antennas mounted on a host spacecraft

We are in the fortunate situation that the GPS signal contains the position coordinates of the GPS satellite in a known reference system. Hence all GPS directions $v_{j}$ are known on board, and all angles $\alpha_{i j}$ between them can be computed directly via $\cos \alpha_{i j}=\left\langle v_{i}, v_{j}\right\rangle$. The existing vector-based approaches do not make use of the fact that the constellation of antenna baselines and the constellation of GPS directions are both known and that only the link between these two constellations needs to be determined. Instead, the above measurement equations are written in the form $\left\langle v_{i}, u_{j}\right\rangle=m_{i j}\left(\lambda / U_{j}\right)+\Phi_{i j} / U_{j}$ where the unknowns are the integers $m_{i j}$ and the coordinates of the vectors $u_{j}$; i.e., there are altogether $k \ell$ scalar equations for $3 \ell+k \ell$ unknowns. This system of equations is underdetermined, and some best-fit approach has to be taken by scanning through an often astronomical number of potential combinations of integers $m_{i j}$. Those existing 
algorithms which do not make use of kinematical considerations look for unlikely combinations and computational simplifications which reduce the work per single scan to a minimum. Nevertheless, there is always a need to go through many thousand cases per determination. This is where the goniometric approach we are going to present leads to a significant reduction of the computational load.

\section{Preliminaries}

In this section we introduce the basic ingredients which are needed to extend spherical trigonometry to arbitrary dimensions: vector products, covectors, and Gramians. The setting is a vector space $V$ of finite dimension $n$ over $\mathbb{K}=\mathbb{R}$ or $\mathbb{C}$, equipped with an inner product $\langle\cdot, \cdot\rangle$ (which allows us to measure lengths and angles) and a determinant function (which allows us to distinguish between different orientations). Here an inner product is a mapping $V \times V \rightarrow \mathbb{K}$ which is skew-symmetric in the sense that $\langle w, v\rangle=\overline{\langle v, w\rangle}$ for all $v, w \in V$, sesquilinear in the sense that $\left\langle\sum_{i} r_{i} v_{i}, \sum_{j} s_{j} w_{j}\right\rangle=\sum_{i, j} r_{i} \overline{s_{j}}\left\langle v_{i}, w_{j}\right\rangle$ for all $r_{i}, s_{j} \in \mathbb{K}$ and all $v_{i}, w_{j} \in V$ and positive definite in the sense that $\langle v, v\rangle>0$ for all $v \in V \backslash\{0\}$, whereas a determinant function is a mapping det: $V^{n} \rightarrow \mathbb{K}$ which is not identically zero and which is alternating in the sense that $\operatorname{det}\left(v_{\sigma(1)}, \ldots, v_{\sigma(n)}\right)=$ $\operatorname{sign}(\sigma) \operatorname{det}\left(v_{1}, \ldots, v_{n}\right)$ for all $v_{i} \in V$ and all elements $\sigma \in \operatorname{Sym}_{n}$ of the symmetric group on $n$ elements. We start with a short discussion of vector products (cf. [5]).

Definition 3.1 Let $V$ be an $n$-dimensional vector space over $\mathbb{K}$ with an inner product $\langle\cdot, \cdot\rangle$ and a determinant function det. The vector product of $n-1$ vectors $v_{1}, \ldots, v_{n-1} \in V$ is defined to be the unique vector $v_{1} \times \cdots \times v_{n-1}$ such that for all $u \in V$ the equation $\left\langle u, v_{1} \times \cdots \times v_{n-1}\right\rangle=\operatorname{det}\left(u, v_{1}, \ldots, v_{n-1}\right)$ holds.

The basic properties of the vector product are given in the following proposition. (Recall that the classical adjoint $\operatorname{adj}(A)$ of an $(n \times n)$-matrix $A$ is the $(n \times n)$-matrix whose $i j$-entry is $(-1)^{i+j} \operatorname{det} A_{j i}$ where $A_{j i}$ is obtained from $A$ by striking out the $j$-th row and the $i$-th column.)

Proposition 3.2 Let $V$ be an n-dimensional vector space over $\mathbb{K}$ with an inner product $\langle\cdot, \cdot\rangle$ and a determinant function det and let $v_{1}, \ldots, v_{n-1} \in V$.

(a) The vector product $v_{1} \times \cdots \times v_{n-1}$ is orthogonal to each of the vectors $v_{i}$ and depends skew-linearly on each of its arguments.

(b) We have $v_{1} \times \cdots \times v_{n-1}=0$ if and only if $v_{1}, \ldots, v_{n-1}$ are linearly dependent.

(c) We have $\left\langle v_{1}, v_{2} \times \cdots \times v_{n}\right\rangle=(-1)^{n-1}\left\langle v_{n}, v_{1} \times \cdots \times v_{n-1}\right\rangle$ for all vectors $v_{n}$.

(d) If $\sigma \in \operatorname{Sym}_{n-1}$ then $v_{\sigma(1)} \times \cdots \times v_{\sigma(n-1)}=\operatorname{sign}(\sigma)\left(v_{1} \times \cdots \times v_{n-1}\right)$.

(e) If $T: V \rightarrow V$ is an endomorphism of $V$ and if adj denotes the formation of the classical adjoint then $\left(T v_{1}\right) \times \cdots \times\left(T v_{n-1}\right)=\operatorname{adj}\left(T^{\star}\right)\left(v_{1} \times \cdots \times v_{n-1}\right)$.

(f) If $T: V \rightarrow V$ is unitary then $T\left(v_{1} \times \cdots \times v_{n-1}\right)=(\operatorname{det} T) \cdot\left(T v_{1}\right) \times \cdots \times\left(T v_{n-1}\right)$. 
Proof. Parts (a) through (d) are mere restatements of properties of the determinant function. To prove (e) we note that, since both sides of the desired equation depend continuously on $T$ and since every endomorphism of $V$ can be obtained as a limit of isomorphisms, we may assume that $T$ is invertible. For all $u \in V$ we then have

$$
\begin{aligned}
& \left\langle u,\left(T v_{1}\right) \times \cdots \times\left(T v_{n-1}\right)\right\rangle=\operatorname{det}\left(u, T v_{1}, \ldots, T v_{n-1}\right) \\
& =(\operatorname{det} T) \cdot \operatorname{det}\left(T^{-1} u, v_{1}, \ldots, v_{n-1}\right)=(\operatorname{det} T) \cdot\left\langle T^{-1} u, v_{1} \times \cdots \times v_{n-1}\right\rangle \\
& =(\operatorname{det} T) \cdot\left\langle u,\left(T^{-1}\right)^{\star}\left(v_{1} \times \cdots \times v_{n-1}\right)\right\rangle=\left\langle u,(\overline{\operatorname{det} T}) \cdot\left(T^{-1}\right)^{\star}\left(v_{1} \times \cdots \times v_{n-1}\right)\right\rangle \\
& =\left\langle u,\left(\operatorname{det} T^{\star}\right)\left(T^{\star}\right)^{-1}\left(v_{1} \times \cdots \times v_{n-1}\right)\right\rangle=\left\langle u, \operatorname{adj}\left(T^{\star}\right)\left(v_{1} \times \cdots \times v_{n-1}\right)\right\rangle,
\end{aligned}
$$

which gives the claim. If $T$ is unitary then $\operatorname{adj}\left(T^{\star}\right)=\left(\operatorname{det} T^{\star}\right)\left(T^{\star}\right)^{-1}=(\operatorname{det} T)^{-1} T$; hence (f) is an immediate consequence of (e).

We now define, for any ordered family of $n$ vectors in $V$, an ordered set of covectors or dual vectors which, in the case of a vector space basis, represents a consistently oriented family of normal vectors of the hyperplanes spanned by any $n-1$ of the $n$ original vectors.

Definition 3.3 Let $V$ be an $n$-dimensional vector space over $\mathbb{K}$ with an inner product $\langle\cdot, \cdot\rangle$ and a vector product. Given $\left(v_{1}, \ldots, v_{n}\right) \in V^{n}$ we define the covectors $\left(w_{1}, \ldots, w_{n}\right) \in$ $V^{n}$ of $\left(v_{1}, \ldots, v_{n}\right)$ via $w_{i}:=(-1)^{i-1} v_{1} \times \cdots \times\left[v_{i}\right] \times \cdots \times v_{n}$ where the bracket $[\cdot]$ denotes the omission of the element enclosed.

The basic properties of covectors are given in the following proposition. (By the rank of a family of vectors we mean the dimension of the vector space spanned by these vectors.)

\section{Proposition 3.4}

(a) We have $\left\langle v_{i}, w_{j}\right\rangle=\delta_{i j} \cdot \operatorname{det}\left(v_{1}, \ldots, v_{n}\right)$ for all $1 \leq i, j \leq n$.

(b) If $\left(v_{1}, \ldots, v_{n}\right)$ is a basis of $V$ and if $\left(\widehat{v}_{1}, \ldots, \widehat{v}_{n}\right)$ is the dual basis with respect to the given inner product in the sense that $\left\langle v_{i}, \widehat{v}_{j}\right\rangle=\delta_{i j}$ for all $i, j$, then $\widehat{v}_{i}=$ $w_{i} / \overline{\operatorname{det}\left(v_{1}, \ldots, v_{n}\right)}$ for $1 \leq i \leq n$.

(c) The rank of $\left(w_{1}, \ldots, w_{n}\right)$ is given by

$$
\operatorname{rank}\left(w_{1}, \ldots, w_{n}\right)= \begin{cases}n & \text { if } \operatorname{rank}\left(v_{1}, \ldots, v_{n}\right)=n, \\ 1 & \text { if } \operatorname{rank}\left(v_{1}, \ldots, v_{n}\right)=n-1, \\ 0 & \text { if } \operatorname{rank}\left(v_{1}, \ldots, v_{n}\right)<n-1 .\end{cases}
$$

(d) We have $w_{1} \times \cdots \times\left[w_{i}\right] \times \cdots \times w_{n}=(-1)^{i-1} \operatorname{det}\left(v_{1}, \ldots, v_{n}\right)^{n-2} \cdot v_{i}$.

Proof. (a) If $i \neq j$ then $\left\langle v_{i}, w_{j}\right\rangle=0$ by Proposition 3.2(a). Moreover, the definition of the vectors $w_{i}$ shows that $\left\langle v_{i}, w_{i}\right\rangle=(-1)^{i-1} \operatorname{det}\left(v_{i}, v_{1}, \ldots, v_{i-1}, v_{i+1}, \ldots, v_{n}\right)=$ $\operatorname{det}\left(v_{1}, \ldots, v_{n}\right)$.

(b) If we take $\widehat{v}_{i}:=w_{i} / \overline{\operatorname{det}\left(v_{1}, \ldots, v_{n}\right)}$ as a definition of $\widehat{v}_{i}$, then part (a) implies that $\left\langle v_{i}, \widehat{v}_{j}\right\rangle=\left\langle v_{i}, w_{j}\right\rangle / \operatorname{det}\left(v_{1}, \ldots, v_{n}\right)=\delta_{i j}$; this yields the claim. 
(c) Let $r:=\operatorname{rank}\left(v_{1}, \ldots, v_{n}\right)$. If $r=n$ the claim follows from part (b). If $r<n$ we may assume without loss of generality that a maximal independent set of the vectors $v_{i}$ is contained in $\left\{v_{1}, \ldots, v_{n-1}\right\}$; then Proposition 3.2(b) shows that $w_{n} \neq 0$ if $r=n-1$ whereas $w_{n}=0$ if $r<n-1$. Now there is an equation of the form $v_{n}=\sum_{j=1}^{n-1} \lambda_{j} v_{j}$, which implies that

$$
\begin{aligned}
w_{i} & =(-1)^{i-1} v_{1} \times \cdots \times\left[v_{i}\right] \times \cdots \times v_{n} \\
& =\sum_{j=1}^{n-1} \overline{\lambda_{j}}(-1)^{i-1} v_{1} \times \cdots \times\left[v_{i}\right] \times \cdots \times v_{j} \\
& =\overline{\lambda_{i}}(-1)^{i-1} v_{1} \times \cdots \times\left[v_{i}\right] \times \cdots \times v_{i} \\
& =\overline{\lambda_{i}}(-1)^{i-1}(-1)^{n-1-i} v_{1} \times \cdots \times v_{i} \times \cdots \times v_{n-1} \\
& =\overline{\lambda_{i}}(-1)^{n} v_{1} \times \cdots \times v_{i} \times \cdots \times v_{n-1}=-\overline{\lambda_{i}} w_{n}
\end{aligned}
$$

for $1 \leq i \leq n-1$. This shows that a maximal independent subset of the vectors $w_{i}$ is given by $\left\{w_{n}\right\}$ if $r=n-1$ and by the empty set if $r<n-1$.

(d) The claim is an immediate consequence of part (c) and Proposition 3.2(b) if $v_{1}, \ldots, v_{n}$ are linearly dependent. If $v_{1}, \ldots, v_{n}$ are linearly independent, we use parts (a) and (b) and the fact that taking the dual basis twice gives back the original basis to see that

$$
\begin{aligned}
v_{i}=\widehat{\widehat{v}}_{i} & =\frac{(-1)^{i-1} \widehat{v}_{1} \times \cdots \times\left[\widehat{v}_{i}\right] \times \cdots \times \widehat{v}_{n}}{\overline{\operatorname{det}\left(\widehat{v}_{1}, \ldots, \widehat{v}_{n}\right)}} \\
& =(-1)^{i-1} \operatorname{det}\left(v_{1}, \ldots, v_{n}\right) \widehat{v}_{1} \times \cdots \times\left[\widehat{v}_{i}\right] \times \cdots \times \widehat{v}_{n} \\
& =(-1)^{i-1} \frac{\operatorname{det}\left(v_{1}, \ldots, v_{n}\right)}{\operatorname{det}\left(v_{1}, \ldots, v_{n}\right)^{n-1}} w_{1} \times \cdots \times\left[w_{i}\right] \times \cdots \times w_{n}
\end{aligned}
$$

where we used the skew-linearity of the vector product in each of its arguments and the fact that $\operatorname{det}\left(v_{1}, \ldots, v_{n}\right) \overline{\operatorname{det}\left(\widehat{v}_{1}, \ldots, \widehat{v}_{n}\right)}=1$.

The last prerequisite for our discussion of goniometry is the formation of Gramians.

Definition 3.5 Let $V$ be an $n$-dimensional vector space over $\mathbb{K}$ with an inner product $\langle\cdot, \cdot\rangle$. The Gramian of $n$ vectors $v_{1}, \ldots, v_{n} \in V$ is the $(n \times n)$-matrix

$$
G\left(v_{1}, \ldots, v_{n}\right):=\left[\begin{array}{ccc}
\left\langle v_{1}, v_{1}\right\rangle & \ldots & \left\langle v_{1}, v_{n}\right\rangle \\
\vdots & & \vdots \\
\left\langle v_{n}, v_{1}\right\rangle & \cdots & \left\langle v_{n}, v_{n}\right\rangle
\end{array}\right] .
$$

It is easy to establish (and well-known; see [9], pp. 406-408) that a matrix $G \in \mathbb{K}^{n \times n}$ is a Gramian if and only if it is self-adjoint and positive semidefinite. It is positive definite (i.e., invertible) if and only if the underlying vectors $v_{1}, \ldots, v_{n}$ are linearly independent. The basic properties of Gramians are summarized in the following proposition. 
Proposition 3.6 Let $V$ be an n-dimensional vector space over $\mathbb{K}$ with an inner product $\langle\cdot, \cdot\rangle$ and a determinant function det, let $v_{1}, \ldots, v_{n} \in V$ be arbitrary vectors and let $\left(w_{1}, \ldots, w_{n}\right)$ be the covectors of $\left(v_{1}, \ldots, v_{n}\right)$. Then the following conditions hold:

(a) $G\left(\lambda v_{1}, \ldots, \lambda v_{n}\right)=|\lambda|^{2} G\left(v_{1}, \ldots, v_{n}\right)$ for all $\lambda \in \mathbb{K}$;

(b) $G\left(T v_{1}, \ldots, T v_{n}\right)=G\left(v_{1}, \ldots, v_{n}\right)$ whenever $T: V \rightarrow V$ is unitary;

(c) $\operatorname{rank} G\left(v_{1}, \ldots, v_{n}\right)=\operatorname{rank}\left(v_{1}, \ldots, v_{n}\right)$;

(d) $\operatorname{det} G\left(v_{1}, \ldots, v_{n}\right)=\left|\operatorname{det}\left(v_{1}, \ldots, v_{n}\right)\right|^{2}$;

(e) $G\left(w_{1}, \ldots, w_{n}\right)=\operatorname{adj}\left(G\left(v_{1}, \ldots, v_{n}\right)\right)$;

(f) if $v_{1}, \ldots, v_{n}$ are linearly independent then $G\left(v_{1}, \ldots, v_{n}\right)^{-1}=G\left(\widehat{v}_{1}, \ldots, \widehat{v}_{n}\right)^{\star}$ where $\widehat{v}_{i}=w_{i} / \overline{\operatorname{det}\left(v_{1}, \ldots, v_{n}\right)}$.

Proof. Parts (a) and (b) follow immediately from the definition of a Gramian; for (c) we refer to [9], Theorem 7.2.10. To prove (d) we express $v_{1}, \ldots, v_{n}$ in coordinates with respect to any orthonormal basis of $V$, form the matrix $A$ whose columns are these coordinate representations, and observe that $G=G\left(v_{1}, \ldots, v_{n}\right)$ equals $A^{\star} A$; hence $\operatorname{det} G=\operatorname{det}\left(A A^{\star}\right)=(\operatorname{det} A)(\overline{\operatorname{det} A})=|\operatorname{det} A|^{2}=\left|\operatorname{det}\left(v_{1}, \ldots, v_{n}\right)\right|^{2}$. To prove (e) we observe that both sides of the desired equation depend continuously on $v_{1}, \ldots, v_{n}$; hence, since an arbitrary $n$-tuple of vectors can be obtained as a limit of linearly independent $n$ tuples, we may assume without loss of generality that $v_{1}, \ldots, v_{n}$ are linearly independent. In this case $A^{\star}=\operatorname{det}\left(v_{1}, \ldots, v_{n}\right) B^{-1}$ where $B$ is the matrix with columns $w_{1}, \ldots, w_{n}$, because $A^{\star} B=\left(\left\langle v_{i}, w_{j}\right\rangle\right)_{i, j}=\operatorname{det}\left(v_{1}, \ldots, v_{n}\right) \mathbf{1}$ by Proposition 3.4(a). Consequently, $\left(A^{\star} A\right)^{-1}=\left|\operatorname{det}\left(v_{1}, \ldots, v_{n}\right)\right|^{-2} B^{\star} B$, i.e.,

$$
G\left(v_{1}, \ldots, v_{n}\right)^{-1}=\left|\operatorname{det}\left(v_{1}, \ldots, v_{n}\right)\right|^{-2} G\left(w_{1}, \ldots, w_{n}\right) .
$$

Together with part (d) this shows that

$$
G\left(w_{1}, \ldots, w_{n}\right)=\operatorname{det} G\left(v_{1}, \ldots, v_{n}\right) G\left(v_{1}, \ldots, v_{n}\right)^{-1}=\operatorname{adj}\left(G\left(v_{1}, \ldots, v_{n}\right)\right) .
$$

Finally, using part (a) and formula $(\star)$, we find that

$$
G\left(v_{1}, \ldots, v_{n}\right)^{-1}=G\left(\frac{w_{1}}{\overline{\operatorname{det}\left(v_{1}, \ldots, v_{n}\right)}}, \ldots, \frac{w_{n}}{\overline{\operatorname{det}\left(v_{1}, \ldots, v_{n}\right)}}\right)=G\left(\widehat{v}_{1}, \ldots, \widehat{v}_{n}\right) .
$$

\section{Goniometry}

In this section we start discussing goniometry proper. We define goniometric sine and cosine functions and derive the higher-dimensional analogues of the well-known formulas of spherical trigonometry.

Definition 4.1 A goniometric cosine is a pair $(\mathrm{gcos}, A)$ where $A \subseteq \mathbb{K}$ is a subset of $\mathbb{K}$ selected such that gcos is a bijection from $A$ onto $\mathbb{U}:=\{z \in \mathbb{K}|| z \mid \leq 1\}$. The corresponding goniometric sine is the nonnegative real-valued function gsin defined by $\operatorname{gsin} \alpha:=\sqrt{1-|g \cos \alpha|^{2}}$. A goniometric space is a finite-dimensional vector space over $\mathbb{K}$ equipped with an inner product and a goniometric cosine. An oriented goniometric space is a goniometric space equipped with a determinant function. 
Formally, a goniometric space is a quadruplet $(V,\langle\cdot, \cdot\rangle, \mathrm{gcos}, A)$ whereas an oriented goniometric space is a quintuplet $(V,\langle\cdot, \cdot\rangle$, det, gcos, $A)$. We simply speak of a goniometric space $V$ or an oriented goniometric space $V$, assuming the other data as being given.

Definition 4.2 Let $v_{1}, \ldots, v_{k}$ be vectors in a goniometric space over $\mathbb{K}$. The goniometric angles $\alpha_{i j}, \alpha^{i j}$ and $\alpha_{i}$ (where $1 \leq i, j \leq k$ ) associated with these vectors, which will be referred to as subscript angles, superscript angles and normal arcs, respectively, are defined by the equations

$$
\begin{aligned}
\left\langle v_{i}, v_{j}\right\rangle & =\left\|v_{i}\right\|\left\|v_{j}\right\| \mathrm{g} \cos \alpha_{i j}, \\
\left\langle w_{i}, w_{j}\right\rangle & =\left\|w_{i}\right\|\left\|w_{j}\right\| \mathrm{g} \cos \alpha^{i j} \\
\left\langle v_{i}, w_{i}\right\rangle & =\left\|v_{i}\right\|\left\|w_{i}\right\| \mathrm{g} \cos \alpha_{i}
\end{aligned}
$$

where the covectors $w_{i}$ are formed within the subspace spanned by $v_{1}, \ldots, v_{k}$.

Remark 4.3 For $V=\mathbb{R}^{3}$ the goniometric subscript angles $\alpha_{i j}$ correspond to arcs on the unit sphere whereas the goniometric superscript angles $\alpha^{i j}$ correspond to the usual dihedral angles of spherical trigonometry if gcos is taken as the conventional cosine function. The goniometric angles $\alpha_{i}$ are normally not considered in spherical trigonometry; we call them normal arcs because, given $v_{1}, v_{2}, v_{3}$ with covectors $w_{1}=$ $v_{2} \times v_{3}, w_{2}=v_{3} \times v_{1}$ and $w_{3}=v_{1} \times v_{2}$, the angle $\alpha_{i}$ represents an arc connecting the unit vector $v_{i} /\left\|v_{i}\right\|$ with a unit normal of the plane spanned by $v_{j}$ and $v_{k}$. As will be discussed later, the sign convention adopted in Definition 3.3 will lead to differences in sign between our goniometric functions and the trigonometric functions usually used in spherical trigonometry.

In the next theorem we essentially show that the goniometric angles and norms of the covectors associated with an $n$-tuple of vectors are geometric invariants, where the geometry is the one derived from the inner product.

Theorem 4.4 Let $\alpha_{i j}, \alpha^{i j}$ and $\alpha_{i}$ be the goniometric angles associated with an n-tuple $\left(v_{1}, \ldots, v_{n}\right)$ of vectors. Then the angles $\alpha_{i j}$ and $\alpha^{i j}$ are invariant under arbitrary unitary transformations whereas the angles $\alpha_{i}$ are invariant under unitary transformations of determinant 1 .

Proof. Given the vectors $v_{1}, \ldots, v_{n}$ and their covectors $w_{i}=(-1)^{i-1} v_{1} \times \cdots \times\left[v_{i}\right] \times$ $\cdots \times v_{n}$ and a unitary transformation $T: V \rightarrow V$, we have to show that the vectors $v_{i}^{\star}:=T v_{i}$ yield the same goniometric angles as the original vectors $v_{i}$. Clearly, $\left\langle v_{i}^{\star}, v_{j}^{\star}\right\rangle=\left\langle T v_{i}, T v_{j}\right\rangle=\left\langle v_{i}, v_{j}\right\rangle$ for all $i, j$ which shows that the subscript angles are geometric invariants. Moreover, $w_{i}^{\star}=(-1)^{i-1} v_{1}^{\star} \times \cdots \times\left[v_{i}^{\star}\right] \times \cdots \times v_{n}^{\star}=(\operatorname{det} T)^{-1} T w_{i}$ due to Proposition 3.2(f) which implies that $\left\langle w_{i}^{\star}, w_{j}^{\star}\right\rangle=|\operatorname{det} T|^{-2}\left\langle T w_{i}, T w_{j}\right\rangle=\left\langle w_{i}, w_{j}\right\rangle$ so that, in particular, lengths of covectors are preserved. Finally, also according to Proposition 3.2(f), we have $\left\langle v_{i}^{\star}, w_{i}^{\star}\right\rangle=\left\langle T v_{i},(\operatorname{det} T)^{-1} T w_{i}\right\rangle=(\operatorname{det} T)\left\langle v_{i}, w_{i}\right\rangle$.

The following result generalizes the well-known trigonometric formulas for spherical triangles. 
Theorem 4.5 Let $v_{1}, \ldots, v_{n}$ be linearly independent unit vectors and let $\left(w_{1}, \ldots, w_{n}\right)$ be the corresponding set of covectors. Moreover, let $G:=G\left(v_{1}, \ldots, v_{n}\right)=\left(\operatorname{gcos} \alpha_{i j}\right)_{i, j}$ and $H:=G\left(w_{1} /\left\|w_{1}\right\|, \ldots, w_{n} /\left\|w_{n}\right\|\right)=\left(\operatorname{gcos} \alpha^{i j}\right)_{i, j}$. Then the following goniometric formulas hold:

(a) $\operatorname{gcos} \alpha^{i j} \sqrt{\operatorname{det} G_{i i}} \sqrt{\operatorname{det} G_{j j}}=(-1)^{i+j} \operatorname{det} G_{j i}$ (cosine rule for superscript angles);

(b) $\frac{\sqrt{\operatorname{det} H_{11}}}{\sqrt{\operatorname{det} G_{11}}}=\frac{\sqrt{\operatorname{det} H_{22}}}{\sqrt{\operatorname{det} G_{22}}}=\cdots=\frac{\sqrt{\operatorname{det} H_{n n}}}{\sqrt{\operatorname{det} G_{n n}}}$ (sine rule) ;

(c) $\operatorname{gcos} \alpha_{i j} \sqrt{\operatorname{det} H_{i i}} \sqrt{\operatorname{det} H_{j j}}=(-1)^{i+j} \operatorname{det} H_{j i}$ (cosine rule for subscript angles);

(d) $\operatorname{gcos} \alpha_{i} \sqrt{\operatorname{det} G_{i i}}=\operatorname{det}\left(v_{1}, \ldots, v_{n}\right)$ (normal arc equations).

Proof. If we let $r_{i}:=(-1)^{i-1} \widetilde{w_{1}} \times \cdots \times\left[\widetilde{w}_{i}\right] \times \cdots \times \widetilde{w_{n}}$ where $\widetilde{w}_{i}:=w_{i} /\left\|w_{i}\right\|$, then Proposition 3.6(e) yields the equations

and

$$
\left\langle w_{i}, w_{j}\right\rangle=(-1)^{i+j} \operatorname{det} G_{j i}
$$

$$
\left\langle r_{i}, r_{j}\right\rangle=(-1)^{i+j} \operatorname{det} H_{j i}
$$

which will be used in the proof. Part (a) follows immediately from the equation gcos $\alpha^{i j}=$ $\left\langle w_{i}, w_{j}\right\rangle /\left(\left\|w_{i}\right\|\left\|w_{j}\right\|\right)$ by using Formula (1) and the fact that $\left\|w_{i}\right\|=\sqrt{\operatorname{det} G_{i i}}$ for all $i$ which, in turn, follows from Formula (1) by choosing $j=i$. For part (b) we use Proposition 3.4(d) to find that

$$
\begin{aligned}
\left\|r_{i}\right\| & =\left\|\frac{w_{1}}{\left\|w_{1}\right\|} \times \cdots \times\left[\frac{w_{i}}{\left\|w_{i}\right\|}\right] \times \cdots \times \frac{w_{n}}{\left\|w_{n}\right\|}\right\| \\
& =\frac{\left\|w_{1} \times \cdots \times\left[w_{i}\right] \times \cdots \times w_{n}\right\|}{\left\|w_{1}\right\| \cdots\left[\left\|w_{i}\right\|\right] \cdots\left\|w_{n}\right\|} \\
& =\frac{\left|\operatorname{det}\left(v_{1}, \ldots, v_{n}\right)\right|^{n-2}}{\left\|w_{1}\right\| \cdots\left\|w_{n}\right\|} \cdot\left\|w_{i}\right\| ;
\end{aligned}
$$

hence $\left\|r_{i}\right\| /\left\|w_{i}\right\|=\left\|r_{j}\right\| /\left\|w_{j}\right\|$ for all $i, j$. Now the claim follows from the facts that $\left\|w_{i}\right\|=\sqrt{\operatorname{det} G_{i i}}$ and $\left\|r_{i}\right\|=\sqrt{\operatorname{det} H_{i i}}$ for all $i$. To prove part (c) we use Proposition 3.4(b) and Equation (2) above to find that

$$
\operatorname{gcos} \alpha_{i j}=\frac{\left\langle v_{i}, v_{j}\right\rangle}{\left\|v_{i}\right\|\left\|v_{j}\right\|}=\frac{\left\langle r_{i}, r_{j}\right\rangle}{\left\|r_{i}\right\|\left\|r_{j}\right\|}=\frac{(-1)^{i+j} \operatorname{det} H_{j i}}{\sqrt{\operatorname{det} H_{i i}} \sqrt{\operatorname{det} H_{j j}}} .
$$

Finally, using Proposition 3.4(a) and Equation (1) above we find that $\operatorname{det}\left(v_{1}, \ldots, v_{n}\right)=$ $\left\langle v_{i}, w_{i}\right\rangle=\left\|w_{i}\right\| \mathrm{g} \cos \alpha_{i}=\sqrt{\operatorname{det} G_{i i}} \mathrm{~g} \cos \alpha_{i}$, which is part (d).

Remark 4.6 For $n=3$ the cosine rule for superscript angles gives rise to the formulas

$$
\begin{aligned}
& \mathrm{g} \cos \alpha^{12} \mathrm{~g} \sin \alpha_{13} \mathrm{~g} \sin \alpha_{23}=-\mathrm{g} \cos \alpha_{12}+\mathrm{g} \cos \alpha_{13} \mathrm{~g} \cos \alpha_{32}, \\
& \mathrm{~g} \cos \alpha^{13} \mathrm{~g} \sin \alpha_{12} \mathrm{~g} \sin \alpha_{23}=+\mathrm{g} \cos \alpha_{12} \mathrm{~g} \cos \alpha_{23}-\mathrm{g} \cos \alpha_{13}, \\
& \mathrm{~g} \cos \alpha^{23} \mathrm{~g} \sin \alpha_{13} \mathrm{~g} \sin \alpha_{12}=-\mathrm{g} \cos \alpha_{23}+\mathrm{g} \cos \alpha_{21} \mathrm{~g} \cos \alpha_{13},
\end{aligned}
$$


while the cosine rule for subscript angles yields exactly the same formulas with superscript and subscript angles exchanged. To compare these equations with the usual formulas of spherical trigonometry we note that, in view of our sign conventions in Definition 3.3, the trigonometric dihedral angles $\beta^{i j}$ differ from the corresponding goniometric angles $\alpha^{i j}$ in such a way that $\operatorname{gcos} \alpha^{i j}=-\cos \beta^{i j}$. Using the conventional cosine function as our goniometric cosine, this means that $\beta^{i j}=\pi-\alpha^{i j}$ for all indices $i, j$. As a consequence, the above equations are the same as the ones used in spherical trigonometry. The same holds true for the sine rule, which takes the form

$$
\frac{\mathrm{g} \sin \alpha^{12}}{\mathrm{~g} \sin \alpha_{12}}=\frac{\mathrm{g} \sin \alpha^{13}}{\mathrm{~g} \sin \alpha_{13}}=\frac{\mathrm{g} \sin \alpha^{23}}{\mathrm{~g} \sin \alpha_{23}} .
$$

The normal arc equations are best considered in connection with the sine rule; they yield the formulas

$$
\frac{\mathrm{g} \cos \alpha_{1}}{\mathrm{~g} \cos \alpha_{2}}=\frac{\mathrm{g} \sin \alpha_{13}}{\mathrm{~g} \sin \alpha_{23}}=\frac{\mathrm{g} \sin \alpha^{13}}{\mathrm{~g} \sin \alpha^{23}} \quad \text { and } \quad \frac{\mathrm{g} \cos \alpha_{1}}{\mathrm{~g} \cos \alpha_{3}}=\frac{\mathrm{g} \sin \alpha_{12}}{\mathrm{~g} \sin \alpha_{23}}=\frac{\mathrm{g} \sin \alpha^{12}}{\mathrm{~g} \sin \alpha^{23}} .
$$

We also remark that - contrary to conventional wisdom - spherical trigonometry over $\mathbb{R}$ defined by the usual cosine and sine rules applies to angles between 0 and $2 \pi$, as is clearly stated by Darboux ([4], Book 2, pp. 203-208). This feature of spherical trigonometry is not inherent to the rules $(\star)$. It is, however, possible to derive spherical trigonometry from goniometry for $n=3$ over $\mathbb{R}$ by consistently endowing selected goniometric function values in the goniometric sine and cosine rules with minus signs. Such sign endowments are possible as long as we maintain equation integrity with respect to the original equations (in which all gsines are bound to be positive). Keeping the signs of $g \cos \alpha_{12}, g \cos \alpha_{13}, g \cos \alpha_{23}$ fixed, all signs of other goniometric functions can be selected within the limits set by the cosine rule for superscript angles on the one hand, implying $\operatorname{sign}\left\{\mathrm{g} \cos \alpha^{i j} \mathrm{~g} \sin \alpha_{i k} \mathrm{~g} \sin \alpha_{j k}\right\}=$ fixed, and by the sine rule on the other hand, namely $\operatorname{sign}\left\{g \sin \alpha^{12} / g \sin \alpha_{12}\right\}=\operatorname{sign}\left\{g \sin \alpha^{13} / g \sin \alpha_{13}\right\}=\operatorname{sign}\left\{g \sin \alpha^{23} / g \sin \alpha_{23}\right\}$. One can check that in the 16 possible valid sign combinations the integrity of the cosine rule for subscript angles remains unaffected each time. As an arbitrary example we select $\operatorname{gsin} \alpha_{12}<0$ and $g \sin \alpha^{12}>0$, which still leaves four possibilities to satisfy the sine rule. We select, again arbitrarily, gsin $\alpha_{13}>0$ and $g \sin \alpha_{23}>0$; the remaining two gsines are then negative. Hence, the sign of the left-hand side of the cosine rule just given is kept constant by leaving $g \cos \alpha^{12}$ as it is, and replacing $g \cos \alpha^{13}$ and $g \cos \alpha^{23}$ by $-g \cos \alpha^{13}$ and $-\mathrm{g} \cos \alpha^{23}$, respectively.

\section{Goniometric families and constellations}

In this section we present the goniometric facts on which our approach to the above attitude determination problem will be based. Our driving theme is the translation of statements about geometric directions from the language of vectors into the language of angles and vice versa. Specifically, we propose certain minimal sets of goniometric angles as particularly suitable data to parametrize sets of direction vectors modulo unitary transformations. 
Definition 5.1 Let $V$ be an inner product space. A frame of order $k$ (or simply $k$-frame) in $V$ is a $k$-tuple $\left(v_{1}, \ldots, v_{k}\right)$ of unit vectors in $V$. The rank $r$ of such a frame is the dimension of the vector space spanned by $v_{1}, \ldots, v_{k}$, and any $r$ of these vectors which are linearly independent are said to form a skeleton of the frame. A constellation of order $k$ in $V$ is an equivalence class of $k$-frames where two frames $\left(v_{1}, \ldots, v_{k}\right)$ and $\left(v_{1}^{\prime}, \ldots, v_{k}^{\prime}\right)$ are declared to be equivalent if there is a unitary transformation $T: V \rightarrow V$ such that $T v_{i}=v_{i}^{\prime}$ for $1 \leq i \leq k$. If $V$ is oriented then an oriented constellation of order $k$ in $V$ is an equivalence class of $k$-frames in $V$ where two such frames $\left(v_{1}, \ldots, v_{k}\right)$ and $\left(v_{1}^{\prime}, \ldots, v_{k}^{\prime}\right)$ are declared to be equivalent if there is a unitary transformation $T: V \rightarrow V$ of determinant 1 such that $T v_{i}=v_{i}^{\prime}$ for $1 \leq i \leq k$. If $\mathbb{C}$ is the (oriented) constellation of a frame $F$, then $F$ is said to represent or to underly 5 . The rank of a constellation or an oriented constellation is the rank of any of its underlying frames.

Note that for $r<n$ the notions of constellations and oriented constellations coincide, as any unitary transformation defined on a proper subspace of $V$ can be extended to a unitary transformation of all of $V$ having determinant 1 . Hence the concept of oriented constellations makes sense only for sets of unit directions which have full rank.
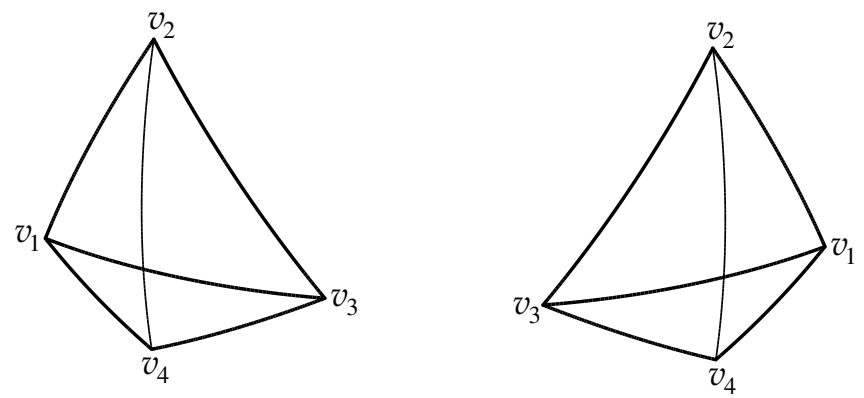

Fig. 2 Two 4-tuples of directions in $\mathbb{R}^{3}$ which represent the same constellation but different oriented constellations

Definition 5.2 A collection $\mathfrak{F}$ of $k(k-1) / 2$ angles $\alpha_{i j}$ (with $1 \leq i<j \leq k$ ) is called a goniometric family of rank $r$ and order $k$ for a goniometric space $V$ if there is a $k$-tuple of unit vectors $\left(v_{1}, \ldots, v_{k}\right)$ of rank $r$ in $V$ such that $g \cos \alpha_{i j}=\left\langle v_{i}, v_{j}\right\rangle$ for all $i<j$. We say that $\mathfrak{\wp}$ is a goniometric family for the $k$-tuple $\left(v_{1}, \ldots, v_{k}\right)$ and that this $k$-tuple is underlying the goniometric family $\mathfrak{F}$.

It will be convenient to also use the angles $\alpha_{i j}$ where $i \geq j$, which are given by $\operatorname{gcos} \alpha_{i j}=\overline{\mathrm{g} \cos \alpha_{j i}}$ and $\mathrm{g} \cos \alpha_{i i}=1$. Note that we defined goniometric families in terms of subscript angles. We could have chosen superscript angles as well, as the cosine rules assure us that we can determine the subscript angles of a family of unit vectors from their superscript angles and vice versa. It would also be possible to deal with goniometric families in which one subscript angle is replaced by the corresponding superscript angle and vice versa, but no good use can be made of "mixed" goniometric families (consisting of both subscript and superscript angles) in reasonable generality. Moreover, in view of 
the application we have in mind, the use of subscript angles is the most natural choice. We first show that there is a simple criterion to establish whether or not a family of angles is a goniometric family.

Theorem 5.3 A family $\mathfrak{F}=\left\{\alpha_{i j} \mid 1 \leq i<j \leq k\right\}$ of $k(k-1) / 2$ angles is a goniometric family of rank $r$ if and only if the matrix

$$
C:=\left[\begin{array}{cccc}
1 & \mathrm{~g} \cos \alpha_{12} & \cdots & \mathrm{g} \cos \alpha_{1 k} \\
\frac{\mathrm{g} \cos \alpha_{12}}{\vdots} & 1 & \cdots & \mathrm{g} \cos \alpha_{2 k} \\
\frac{\vdots}{\mathrm{g} \cos \alpha_{1 k}} & \frac{\ddots}{\mathrm{gcos} \alpha_{2 k}} & \cdots & \vdots
\end{array}\right]
$$

(which we call the characteristic matrix of $\mathfrak{F}$ ) is positive semidefinite and has rank $r$.

Proof. This is a basic textbook fact about Gram matrices; see [9], Theorem 7.2.10 and Corollary 7.2.11.

Next we establish that a goniometric family uniquely determines the underlying frame modulo a unitary transformation. (This is not very surprising from a geometric point of view, because a frame with all angles specified can be visualized as a rigid body, and unitary transformations represent rigid motions.) As a consequence, goniometric families can serve as data which unambiguously characterize constellations.

Theorem 5.4 Two frames (of the same order) in an inner product space $V$ underly the same goniometric family if and only if they represent the same constellation.

Proof. Let $\left(u_{1}, \ldots, u_{k}\right)$ and $\left(w_{1}, \ldots, w_{k}\right)$ be the two frames in question and represent all $u_{i}$ 's and $w_{i}$ 's in coordinates with respect to an arbitrary orthonormal basis of $V$. Denoting by $U=\left(u_{1}|\cdots| u_{k}\right) \in \mathbb{K}^{n \times k}$ and $W=\left(w_{1}|\cdots| w_{k}\right) \in \mathbb{K}^{n \times k}$ the matrices whose columns are these coordinate representations, the statement of the theorem is that $U^{\star} U=W^{\star} W$ if and only if there is a unitary matrix $T \in \mathbb{K}^{n \times n}$ such that $W=T U$. Clearly, if such a $T$ exists then $W^{\star} W=U^{\star} T^{\star} T U=U^{\star} U$. Assume conversely that $W^{\star} W=U^{\star} U$; then $r:=\operatorname{rank} U=\operatorname{rank} U^{\star} U=\operatorname{rank} W^{\star} W=\operatorname{rank} W$. After reindexing we may assume that $\left(u_{1}, \ldots, u_{r}\right)$ forms a skeleton of $\left(u_{1}, \ldots, u_{k}\right)$. Write $U=$ $\left(U_{1} \mid U_{2}\right)$ and $W=\left(W_{1} \mid W_{2}\right)$ where $U_{1}, W_{1} \in \mathbb{K}^{n \times r}$ and $U_{2}, W_{2} \in \mathbb{K}^{n \times(k-r)}$. Each vector $u_{\ell}$ with $\ell>r$ can be uniquely written as $u_{\ell}=\lambda_{1}^{(\ell)} u_{1}+\cdots+\lambda_{r}^{(\ell)} u_{r}$. These equations can be combined to the matrix equation $U_{2}=U_{1} \Lambda$ where $\Lambda:=\left(\lambda_{i}^{(\ell)}\right)_{1 \leq i \leq r, r+1 \leq \ell \leq k}$. Then

$$
\left[\begin{array}{cc}
U_{1}^{\star} U_{1} & U_{1}^{\star} U_{1} \Lambda \\
\Lambda^{\star} U_{1}^{\star} U_{1} & \Lambda^{\star} U_{1}^{\star} U_{1} \Lambda
\end{array}\right]=U^{\star} U=W^{\star} W=\left[\begin{array}{cc}
W_{1}^{\star} W_{1} & W_{1}^{\star} W_{2} \\
W_{2}^{\star} W_{1} & W_{2}^{\star} W_{2}
\end{array}\right]
$$

which implies that $W_{1}^{\star} W_{1}=U_{1}^{\star} U_{1}$ and hence $\operatorname{rank} W_{1}=\operatorname{rank} W_{1}^{\star} W_{1}=\operatorname{rank} U_{1}^{\star} U_{1}=$ rank $U_{1}=r$. This shows that $\left(w_{1}, \ldots, w_{r}\right)$ forms a skeleton of $\left(w_{1}, \ldots, w_{k}\right)$. Hence, in analogy to the decomposition $U_{1}=U_{1} \Lambda$, there is a decomposition $W_{1}=W_{1} \Sigma$ for some $\Sigma \in \mathbb{K}^{r \times(k-r)}$. But then $\left(U_{1}^{\star} U_{1}\right) \Lambda=W_{1}^{\star} W_{2}=W_{1}^{\star} W_{1} \Sigma=\left(U_{1}^{\star} U_{1}\right) \Sigma$ and thus $\Sigma=\Lambda$. (This is just a slick way of saying that the vectors $w_{r+1}, \ldots, w_{k}$ form the same 
linear combinations in terms of $w_{1}, \ldots, w_{r}$ as the ones which $v_{r+1}, \ldots, v_{k}$ form in terms of $v_{1}, \ldots, v_{r}$.) Now apply the Gram-Schmidt orthonormalization procedure to both $U_{1}$ and $W_{1}$; this yields decompositions $U_{1}=P_{0} R$ and $W_{1}=Q_{0} R$ where $P_{0}, Q_{0} \in \mathbb{K}^{n \times r}$ have orthonormal columns and where $R \in \mathbb{K}^{r \times r}$ is upper triangular. (The same matrix $R$ occurs in both equations because the entries of $R$ are obtained in the course of the Gram-Schmidt procedure by computing certain inner products which, by hypothesis, are the same for the $u_{i}$ 's and the $w_{i}$ 's.) Extend $P_{0}$ and $Q_{0}$ to unitary matrices $P, Q \in \mathbb{K}^{n \times n}$ in any way you wish and let $T:=Q P^{-1}=Q P^{\star}$; then $T U=\left(Q P^{\star}\right)\left(P_{0} R \mid P_{0} R \Lambda\right)=$ $\left(Q_{0} R \mid Q_{0} R \Lambda\right)=W$.

The basic fact expressed in Theorem 5.4 has a long history and many interesting applications. (See Theorem 3.1 in [10]; this paper also gives a historical survey and a bibliography.) Some further analysis of the proof is necessary to examine how many (and which) of the entries of $U^{\star} U$ (i.e., which goniometric angles) are necessary to reconstruct $U$ up to unitary transformations. First, we have

$$
\Lambda=\left(U_{1}^{\star} U_{1}\right)^{-1} U_{1}^{\star} U_{2}=\left[\begin{array}{ccc}
1 & \cdots & \mathrm{g} \cos \alpha_{1 r} \\
\vdots & \ddots & \vdots \\
\frac{\operatorname{gcos} \alpha_{1 r}}{} & \cdots & 1
\end{array}\right]^{-1}\left[\begin{array}{ccc}
\mathrm{g} \cos \alpha_{1, r+1} & \cdots & \mathrm{gcos} \alpha_{1 k} \\
\vdots & & \vdots \\
\mathrm{g} \cos \alpha_{r, r+1} & \cdots & \mathrm{g} \cos \alpha_{r k}
\end{array}\right]
$$

which, as is implied by Cramer's Rule, means that we have representations

$$
\lambda_{j}^{(\ell)}=\frac{d_{1 j} \mathrm{~g} \cos \alpha_{1 \ell}+\cdots+d_{r j} \mathrm{~g} \cos \alpha_{r \ell}}{d}
$$

where $d$ is the determinant of the coefficient matrix and where $d_{i j}$ is a polynomial of first degree in each of those expressions gcos $\alpha_{p q}$ and $\overline{\operatorname{gcos} \alpha_{p q}}$ with $1 \leq p<q \leq r$ for which $p \neq i$ and $q \neq j$. Next, the equation $U_{2}^{\star} U_{2}=\Lambda^{\star} U_{1}^{\star} U_{1} \Lambda=U_{2}^{\star} U_{1}\left(U_{1}^{\star} U_{1}\right)^{-1} U_{1}^{\star} U_{2}$ yields gcos $\alpha_{\ell_{1} \ell_{2}}=\sum_{i, j=1}^{r} \overline{\lambda_{i}^{\left(\ell_{1}\right)}} \lambda_{j}^{\left(\ell_{2}\right)}$ gcos $\alpha_{i j}$ for $\ell_{1}, \ell_{2}>r$ which, after plugging in (3) and multiplying by $\bar{d} d$, reads

$$
\bar{d} d \cdot \operatorname{gcos} \alpha_{\ell_{1} \ell_{2}}=\sum_{i, j, p, q=1}^{r} \overline{d_{p i}} d_{q j} \overline{\operatorname{gcos} \alpha_{p \ell_{1}}} \operatorname{gcos} \alpha_{q \ell_{2}} \operatorname{gcos} \alpha_{i j} \quad\left(\ell_{1}, \ell_{2}>r\right) .
$$

Thus the angles $\alpha_{i j}$ with $1 \leq i<j \leq r$ and the angles $\alpha_{m \ell}$ with $1 \leq m \leq r$ and $\ell>r$ determine already all the other angles. However, these angles are not independent; a relation between them is found by letting $\ell_{1}=\ell_{2}=: \ell$ in (4), which yields

$$
\bar{d} d=\sum_{i, j, p, q=1}^{r} \overline{d_{p i}} d_{q j} \overline{\mathrm{g} \cos \alpha_{p \ell}} \mathrm{g} \cos \alpha_{q \ell} \mathrm{g} \cos \alpha_{i j} \quad(\ell>r) ;
$$

this equation can be solved for any of the terms $\operatorname{gcos} \alpha_{\rho \ell}$ for any fixed choice $\rho=\rho(\ell)$. Note that both sides of (4) depend linearly on each of the occurring goniometric cosines and conjugate geometric cosines, so that the equation can be unambiguously solved for each of these terms. On the other hand, solving (5) for the expressions gcos $\alpha_{\rho \ell}$ requires the extraction of square roots. To interpret these results, we introduce the following terminology. (We refer to [11], pp. 151-152 for the concept of algebraic dependence.) 
Definition 5.5 Let $\mathfrak{F}$ be a goniometric family. For any element $\alpha \in \mathfrak{F}$ and any subset $S \subseteq \mathfrak{F}$ we write $\alpha \preceq S$ (read " $\alpha$ depends on $S$ ") if $\operatorname{gcos} \alpha$ and $\overline{\operatorname{gcos} \alpha}$ are algebraically dependent over $\mathbb{Q}$ on $\{\mathrm{g} \cos s, \overline{\mathrm{g} \cos S} \mid s \in S\}$, where each of the elements $\mathrm{g} \cos s$ and $\overline{\mathrm{gcos} S}$ is considered as an indeterminate, i.e., a transcendental over $\mathbb{Q}$. A subset $S \subseteq \mathfrak{F}$ is called

(a) independent if no element $\alpha \in S$ depends on $S \backslash\{\alpha\}$;

(b) generating if every element $\alpha \in \widetilde{F}$ depends on $S$;

(c) a basis if it is both independent and generating.

It is readily checked that, given a goniometric family $\mathfrak{F}$, the relation $\preceq$ is a dependence relation in the general sense of Jacobson (see [11], p. 153), which means that the following axioms are satisfied:

(1) if $\alpha \in S$ then $\alpha \preceq S$;

(2) if $\alpha \preceq S$ then $\alpha \preceq S_{0}$ for some finite subset $S_{0} \subseteq S$;

(3) if $\alpha \preceq S$ and $s \preceq T$ for each $s \in S$ then $\alpha \preceq T$;

(4) if $\alpha \preceq S$ and $\alpha \npreceq S \backslash\left\{s_{0}\right\}$ then $s_{0} \preceq\left(S \backslash\left\{s_{0}\right\}\right) \cup\{\alpha\}$ (exchange axiom).

Therefore ([11], p. 154), a basis of $\mathfrak{\wp}$ necessarily exists (as a basis is the same as a maximal independent subset or a minimal generating subset where maximality and minimality refer to set-theoretical inclusion), and any two bases have the same cardinality (which is just the maximal number of independent angles in $\mathfrak{f}$ ). We are now going to calculate this number.

Theorem 5.6 The maximal number of independent angles within a goniometric family $\mathfrak{r}=\left\{\alpha_{i j} \mid 1 \leq i<j \leq k\right\}$ of rank $r$ is

$$
\Sigma(k, r):=r(r-1) / 2+(r-1)(k-r)=(r-1)(2 k-r) / 2 .
$$

More precisely, if the angles are indexed in such a way that the matrix $\left(\operatorname{gcos} \alpha_{i j}\right)_{1 \leq i, j \leq r}$ has maximal rank $r$ as a submatrix of $\left(g \cos \alpha_{i j}\right)_{1 \leq i, j \leq k}$ and if we choose for each $\ell>r$ an arbitrary element $\rho(\ell) \in\{1, \ldots, r\}$, then the set $S:=\left\{\alpha_{i j} \mid 1 \leq i<j \leq r\right\} \cup\left\{\alpha_{\ell m} \mid\right.$ $\ell>r, 1 \leq m \leq r, m \neq \rho(\ell)\}$ is a maximal independent subset of $\mathfrak{F}$.

Proof. The discussion leading up to Definition 5.5 shows that $S$ is a generating subset of $\mathfrak{F}$. To prove that, on the other hand, $S$ is also independent we show that for an arbitrary prescription of the angles in $S$ (with the only restriction that these angles qualify at all as goniometric angles) an underlying frame can indeed be constructed. The construction, which can be seen as a Gram-Schmidt procedure in reverse, consists in choosing arbitrary elements $\varepsilon_{1}, \ldots, \varepsilon_{k} \in \mathbb{U}=\{\varepsilon \in \mathbb{K}|| \varepsilon \mid=1\}$ and letting $v_{m}:=$ $\left(f_{1}^{(m)}, \ldots, f_{m}^{(m)}, 0, \ldots, 0\right)^{T} \in \mathbb{K}^{r}$ for $1 \leq m \leq r$ where

$$
\begin{aligned}
f_{1}^{(1)} & :=\varepsilon_{1} ; \quad f_{\ell}^{(m)}:=\left(\operatorname{gcos} \alpha_{\ell m}-\sum_{i=1}^{\ell-1} \overline{f_{i}^{(\ell)}} f_{i}^{(m)}\right) / \overline{f_{\ell}^{(\ell)}}(1 \leq \ell \leq m-1), \\
f_{m}^{(m)} & :=\varepsilon_{m} \sqrt{1-\sum_{i=1}^{m-1}\left|f_{i}^{(m)}\right|^{2} .}
\end{aligned}
$$


Next, for $\ell>r$, we choose $\rho(\ell):=r$ for simplicity and define $v_{\ell}:=\left(f_{1}^{(\ell)}, \ldots, f_{r}^{(\ell)}\right)^{T} \in$ $\mathbb{K}^{r}$ where

$$
f_{m}^{(\ell)}:=\frac{\mathrm{g} \cos \alpha_{m \ell}-\sum_{i=1}^{m-1} \overline{f_{i}^{(m)}} f_{i}^{(\ell)}}{\overline{f_{m}^{(m)}}}(1 \leq m \leq r-1), \quad f_{r}^{(\ell)}:=\varepsilon_{\ell} \sqrt{1-\sum_{i=1}^{r-1}\left|f_{i}^{(\ell)}\right|^{2}} . \quad(\star \star)
$$

Comment 5.7 As a consequence of Theorem 5.4, any choices for $\varepsilon_{1}, \ldots, \varepsilon_{r} \in \mathbb{U}$ in step $(\star)$ will result in frames $\left(v_{1}, \ldots, v_{r}\right)$ which represent the same constellation; thus there is no ambiguity in constructing the skeleton of a constellation, and we may as well choose $\varepsilon_{i}:=1$ for $1 \leq i \leq r$. This can be interpreted geometrically by saying that as long as there is a free dimension in which the next unit vector to be constructed can move, all possible choices for this unit vector are equivalent.

For all steps in $(\star \star)$ except for the last one there was no choice; to ensure that gcos $\alpha_{m \ell}=$ $\left\langle v_{m}, v_{\ell}\right\rangle$ we had to choose $f_{m}^{(\ell)}$ the way we did. The last entry must be chosen to make $v_{\ell}$ into a unit vector, and this determines the last entry up to an element $\varepsilon_{\ell} \in \mathbb{U}$, but here different choices for $\varepsilon_{\ell}$ lead to inequivalent frames, i.e., different constellations. Hence once a skeleton is constructed, each new unit vector to be added is only determined up to a factor of absolute value 1 . For $\mathbb{K}=\mathbb{R}$ and $n=3$ this can be easily visualized: given a skeleton $\left(v_{1}, v_{2}, v_{3}\right)$, the specification of $\alpha_{14}$ and $\alpha_{24}$ forces $v_{4}$ to lie in the intersection of two cones around $v_{1}$ and $v_{2}$, which generically consists of two different rays.

The fact that a maximal independent subset of goniometric angles does not fully determine a constellation causes no problem for our application to attitude determination, because for $\mathbb{K}=\mathbb{R}$ the unit group $\mathbb{U}=\{ \pm 1\}$ consists of only two elements, which implies that a maximal independent set of goniometric angles determines a constellation up to a finite number of ambiguities which can easily be dealt with.

Example 5.8 Consider the example in $\mathbb{R}^{3}$ depicted in Fig. 3 in which the angles $\alpha_{12}$, $\alpha_{13}, \alpha_{23}, \alpha_{24}$ and $\alpha_{34}$ are given. Two different values for $\alpha_{14}$ are possible; choosing one of them fixes the constellation up to an orthogonal transformation.

The following remark puts Theorem 5.6 into a more general perspective by interpreting the above result in terms of differential geometry in the case $\mathbb{K}=\mathbb{R}$.

Remark 5.9 Let $\mathbb{K}=\mathbb{R}$. Given an $r$-dimensional subspace $U \subseteq V$, we denote by $\mathbb{S}_{U}:=\{v \in U \mid\|v\|=1\}$ the unit sphere in $U$ (which is an embedded submanifold of dimension $r-1)$. Then $M_{U}:=\left\{\left(v_{1}, \ldots, v_{k}\right) \mid v_{i} \in U,\left\|v_{i}\right\|=1, \operatorname{rank}\left(v_{1}, \ldots, v_{k}\right)=r\right\}$ $\subseteq V \times \cdots \times V$ is an open submanifold of the $k$-fold product of $\mathbb{S}_{U}$ with itself, hence is an embedded submanifold of dimension $k(r-1)$. Let $\mathrm{O}(V)$ be the orthogonal group of $V$. The group $G_{U}:=\left\{g \in \mathrm{O}(V) \mid g \equiv \mathbf{1}\right.$ on $\left.U^{\perp}\right\}$ (which can be identified with $\mathrm{O}(U)$ and hence is a Lie group of dimension $r(r-1) / 2)$ acts on $M_{U}$ via $T \star\left(v_{1}, \ldots, v_{k}\right):=$ $\left(T v_{1}, \ldots, T v_{k}\right)$. Since $G_{U}$ is compact, this action is automatically proper; moreover, it is obvious that no element $T \neq \mathbf{1}$ in $G_{U}$ has a fixed point, which means that the action of 

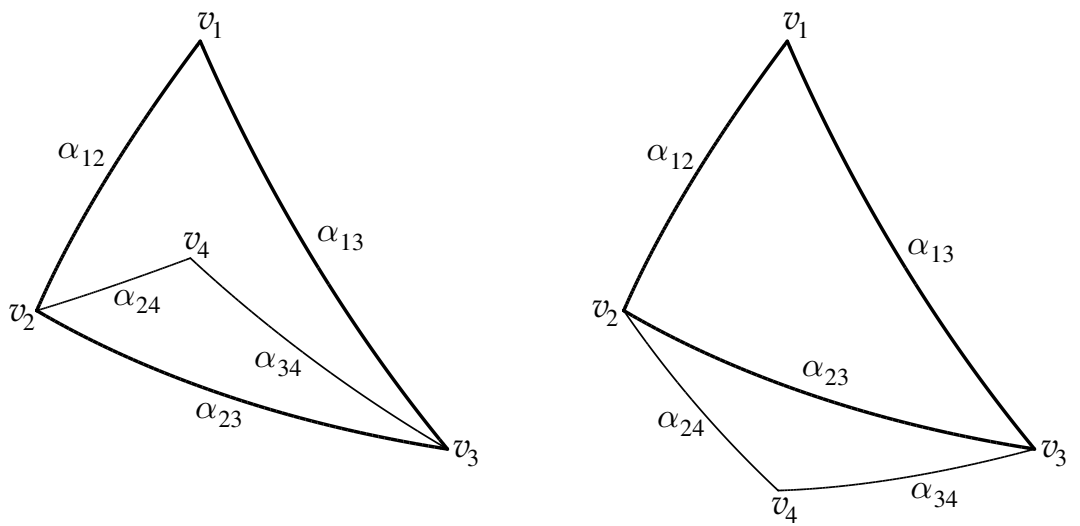

Fig. 3 The two different constellations determined by the angles $\alpha_{12}, \alpha_{13}, \alpha_{23}, \alpha_{24}$ and $\alpha_{34}$

$G_{U}$ on $M_{U}$ is free. Therefore ([7], p. 32, Thm. 1.95) the quotient space $M_{U} / G_{U}$ (which is nothing but the space of all constellations which can be realized by vectors in $U$ ) carries a natural manifold structure for which the canonical projection $\pi: M_{U} \rightarrow M_{U} / G_{U}$ is a submersion. The dimension of $M_{U} / G_{U}$ is

$$
\operatorname{dim}\left(M_{U} / G_{U}\right)=\operatorname{dim} M_{U}-\operatorname{dim} G_{U}=k(r-1)-\frac{r(r-1)}{2}=\frac{1}{2}(r-1)(2 k-r) .
$$

What Theorem 5.6 essentially does is identify a subset of goniometric angles which can be used as local coordinates to parametrize the manifold $M_{U} / G_{U}$.

For the application we have in mind it will be important to find out how many supplementary angles are needed to determine a constellation from two subconstellations; this will be done in the next theorem.

Theorem 5.10 Let $\left(v_{1}, \ldots, v_{k}\right)$ and $\left(u_{1}, \ldots, u_{\ell}\right)$ be two families of unit vectors in $V$. Assume that $\operatorname{rank}\left(v_{1}, \ldots, v_{k}\right)=r_{1}$ and $\operatorname{rank}\left(u_{1}, \ldots, u_{\ell}\right)=r_{2}$. Given maximal independent sets of goniometric angles for the two families separately, one has to add an additional number of

$$
\left(r_{1}+r_{2}\right)\left(r_{3}-1\right)-\sum_{i=1}^{3} r_{i}\left(r_{i}-1\right) / 2
$$

angles to obtain a maximal independent set of goniometric angles for the $(k+\ell)$-tuple $\left(v_{1}, \ldots, v_{k}, u_{1}, \ldots, u_{\ell}\right)$, whose rank is denoted by $r_{3}$.

Proof. The number of additional angles required is $\Sigma\left(r_{1}+r_{2}, r_{3}\right)-\Sigma\left(r_{1}, r_{1}\right)-\Sigma\left(r_{2}, r_{2}\right)$ which, in view of Theorem 5.6, equals $\left(r_{1}+r_{2}\right)\left(r_{3}-1\right)-\sum_{i=1}^{3} r_{i}\left(r_{i}-1\right) / 2$.

Up to this point orientations have played no role in our discussion. However, as alert readers may have expected, we did not include the definition of an oriented constellation in Definition 5.1 for no reason. The reason is that the result of a spacecraft attitude determination is usually not only a constellation, but an oriented constellation. A typical situation is described in the following remark. 
Remark 5.11 Consider a spin-stabilized spacecraft whose spin-axis direction is represented by a unit vector $N$. Optical sensors mounted on such a spacecraft measure the angles $\theta$ and $\beta$ between the spin-axis and the sun direction $S$ on the one hand and between the spin-axis and the earth direction $E$ (also called nadir) on the other hand (so that $\langle S, N\rangle=\cos \theta$ and $\langle E, N\rangle=\cos \beta$ ). The vectors $S$ and $E$ are known in inertial space; consequently, the angle $\gamma$ defined by $\langle S, E\rangle=\cos \gamma$ is also known. The triangle formed by $N, S$ and $E$ (thought of as points on the unit sphere) is then fully determined by the three $\operatorname{arcs} \theta, \beta$ and $\gamma$. However, there are two possible unit vectors $N$ which are consistent with these arcs, which can be distinguished by the sign of the determinant of the triplet $(S, E, N)$. From the early 1970s on, sensor systems have been upgraded as to also measure the dihedral angle $\alpha$ indicated in Fig. 4 below. (We refer to [17], Chapter 10 for general background and to [13], Section 2.1 for one of the earlier implementations on a satellite launched in November 1972.) Knowing $\alpha$ provides us with $\sin \alpha \sin \theta \sin \beta=\langle S \times E, N\rangle=\operatorname{det}(S, E, N)$, thus lifting the ambiguity by fixing the determinant of the triplet $(S, E, N)$. The expression $\sin \alpha \sin \theta \sin \beta$ (which has no immediate physical significance and can be measured only indirectly) is a typical example for the right-hand side of a normal arc equation.

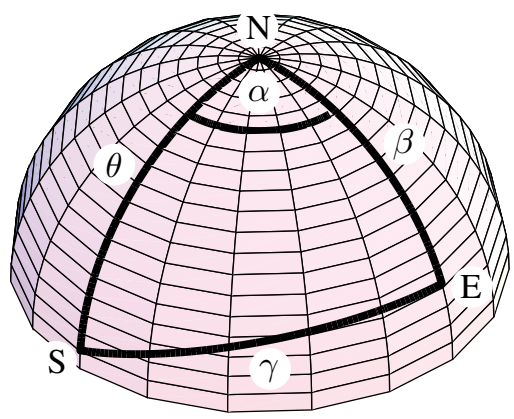

Fig. 4 Representation of spin axis direction $N$, sun direction $S$ and nadir $E$ on the unit sphere

We now show that a goniometric family needs to be augmented by a single normal arc in order to unambiguously represent an oriented constellation. The specification of such a normal arc is equivalent to specifying the determinant of a basis of unit vectors underlying the given goniometric family.

Definition 5.12 A normal goniometric family is a family of angles of the form $\mathfrak{\Im} \cup\left\{\alpha_{*}\right\}$ where $\mathfrak{F}$ is a goniometric family and where $\alpha_{*}$ is a normal arc associated with a family of maximal rank within $\mathfrak{\wp}$.

Theorem 5.13 Two $k$-tuples $\left(v_{1}, \ldots, v_{k}\right) \in V^{k}$ and $\left(v_{1}^{\prime}, \ldots, v_{k}^{\prime}\right) \in V^{k}$ of unit vectors of full rank $n$ are underlying the same normal goniometric family if and only if they represent the same oriented constellation. 
Proof. After re-indexing we may assume that $\left(v_{1}, \ldots, v_{n}\right)$ is a basis of $V$. In view of Theorem 5.4, we only have to show that the unique unitary transformation $T: V \rightarrow V$ satisfying $T v_{i}=v_{i}^{\prime}$ for $1 \leq i \leq n$ preserves one normal arc (hence all normal arcs) if and only if det $T=1$. Now $v_{i}^{\prime}=T v_{i}$ for all $i$ implies $u_{i}^{\prime}=T u_{i}$ for all $i$ and hence $w_{i}^{\prime}=$ $\operatorname{det}\left(v_{1}^{\prime}, \ldots, v_{n}^{\prime}\right) u_{i}^{\prime}=\operatorname{det}\left(T v_{1}, \ldots, T v_{n}\right) T u_{i}=(\operatorname{det} T) \operatorname{det}\left(v_{1}, \ldots, v_{n}\right) T u_{i}=(\operatorname{det} T) T w_{i}$ which is easily seen to imply $\operatorname{gcos} \alpha_{i}^{\prime}=(\operatorname{det} T) \operatorname{gcos} \alpha_{i}$. This gives the claim.

\section{Application to attitude determination}

We now return to the situation described in Section 2. The GPS-directions form a $k$-tuple $\left(v_{1}, \ldots, v_{k}\right)$ which can be assumed to have the full rank 3 , as there are usually enough GPS satellites whose signals can be received by the host spacecraft at any time. The $\ell$ unit vectors representing the baselines are known in the body reference system of the host satellite, but not in the reference system in which the directions $v_{i}$ are given. The rank $r$ of the $\ell$-tuple $\left(u_{1}, \ldots, u_{\ell}\right)$ can be 1,2 or 3 , because the number of antennas on board is normally limited. What is unknown is the link between the $k$-tuple and the $\ell$-tuple which, according to Theorem 5.10, requires a number of supplementary angles which is given by

$$
2 r-\frac{r(r-1)}{2}= \begin{cases}2 & \text { if } r=1, \\ 3 & \text { if } r=2, \\ 3 & \text { if } r=3 .\end{cases}
$$

Now let us explain our approach by means of the example depicted in Fig. 5. (We ignore all technicalities and refer to [6] for details.)
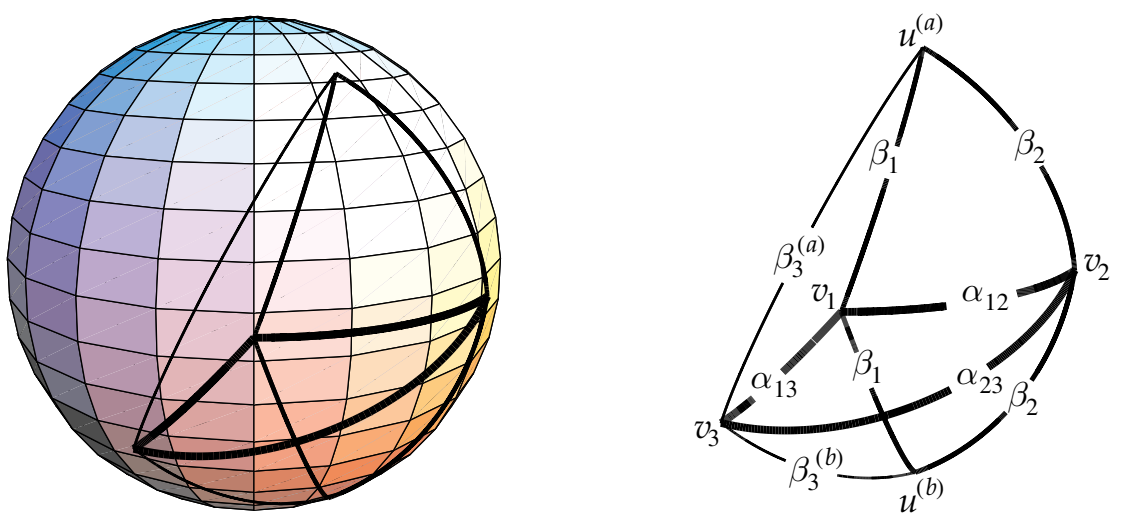

Fig. 5 Typical situation in which the proposed attitude determination algorithm can be applied.

We assume just one baseline $u_{1}$ (so that $r=\ell=1$ ) and four GPS directions $v_{1}, \ldots, v_{4}$ also called Lines Of Sight (LOS). To simplify the notation we write $U:=U_{1}, v_{5}:=u_{1}$ and also $m_{i}:=m_{i 1}$ and $\Phi_{i}:=\Phi_{i 1}$ for $1 \leq i \leq 4$; then the measurement equations read

$$
U \cos \alpha_{i 5}=\Phi_{i}+m_{i} \lambda \quad(\text { where } 1 \leq i \leq 4) .
$$


Since the measurements $\Phi_{i}$ take values in the interval $[0, \lambda)$, this yields the estimates $-(1+U / \lambda)<m_{i} \leq U / \lambda$. (With the typical values of $1 \mathrm{~m}$ for $U$ and $19 \mathrm{~cm}$ for $\lambda$ this means $m_{i} \in\{-6, \ldots, 5\}$ for $1 \leq i \leq 4$.) All angles $\alpha_{15}, \ldots, \alpha_{45}$ are unknown, but, in accordance with Theorem 5.10, any two of them determine the others up to a single ambiguity. For example, if $\alpha_{15}$ and $\alpha_{25}$ are known, then the cosine rule for subscript angles implies

$$
\frac{\cos \alpha_{i 5}-\cos \alpha_{1 i} \cos \alpha_{15}}{\sin \alpha_{1 i} \sin \alpha_{15}}=\cos \alpha_{(1)}^{i 5}= \pm \cos \alpha_{(2)}^{i 5}= \pm \frac{\cos \alpha_{i 5}-\cos \alpha_{2 i} \cos \alpha_{25}}{\sin \alpha_{2 i} \sin \alpha_{25}}
$$

for $i=3,4$, where the sign ambiguity occurring in the calculation of $\cos \alpha^{i 5}$ arises from the fact that $\left(v_{1}, v_{5}, v_{i}\right)$ and $\left(v_{2}, v_{5}, v_{i}\right)$ may or may not have the same orientation. Solving for $\cos \alpha_{i 5}$ yields

$$
\cos \alpha_{i 5}=\frac{\sin \alpha_{1 i} \cos \alpha_{2 i} \sin \alpha_{15} \cos \alpha_{25} \mp \cos \alpha_{1 i} \sin \alpha_{2 i} \cos \alpha_{15} \sin \alpha_{25}}{\sin \alpha_{1 i} \sin \alpha_{15} \mp \sin \alpha_{2 i} \sin \alpha_{25}},
$$

which gives two potential values for the angle $\alpha_{i 5}$. It is thus sufficient to go through the combinations of $m_{1}$ (outer loop) and $m_{2}$ (inner loop) only. For each combination we can compute the two potential values of the angle $\alpha_{35}$ and check for each whether it fits into the measurement equation for the phase difference $\Phi_{3}$ with a valid integer (or a number very close to an integer, allowing for measurement errors). If the fit is satisfactory, the same can be verified for $\Phi_{4}$. If no (near) integer is found one proceeds to the next combination of $m_{1}$ and $m_{2}$. In the absence of outlier measurements one arrives at the result after all combinations of $m_{1}$ and $m_{2}$ have been searched, which amounts to a maximum of 144 cases for the typical configuration of a baseline length of $1 \mathrm{~m}$ and a signal wavelength of $19 \mathrm{~cm}$. Due to measurement errors one can end up with more than one potential solution, just as with vector-based methods. This can be easily resolved if there is more than one baseline, for then solutions can be obtained for every baseline separately, and these solutions can be tested against the known angles amongst the baselines. Let us now compare our method with the brute-force vector approach which is typically used. Writing the known GPS-directions in the form $v_{i}=\left(x_{i}, y_{i}, z_{i}\right)^{T}$ and the unknown base-line direction in the form $u=(x, y, z)^{T}$, the measurement equations take the form $x_{i} x+y_{i} y+z_{i} z-m_{i} \lambda / U=\Phi_{i} / U$ where $1 \leq i \leq 4$. Typically, one goes through all $12^{4}=20736$ possible choices for $\left(m_{1}, m_{2}, m_{3}, m_{4}\right)$, solves for each such choice the overdetermined system

$$
\left[\begin{array}{lll}
x_{1} & y_{1} & z_{1} \\
x_{2} & y_{2} & z_{2} \\
x_{3} & y_{3} & z_{3} \\
x_{4} & y_{4} & z_{4}
\end{array}\right]\left[\begin{array}{l}
x \\
y \\
z
\end{array}\right]=\left[\begin{array}{l}
\left(\Phi_{1}+m_{1} \lambda\right) / U \\
\left(\Phi_{2}+m_{2} \lambda\right) / U \\
\left(\Phi_{3}+m_{3} \lambda\right) / U \\
\left(\Phi_{4}+m_{4} \lambda\right) / U
\end{array}\right]
$$

in the least-squares sense and checks each time whether or not the solution vector $(x, y, z)^{T}$ thus obtained is a unit vector. Despite certain possible simplifications, too technical to be described here, this is a terribly inefficient algorithm. A more sensible 
approach would be to go through all possible choices for $\left(m_{2}, m_{3}, m_{4}\right)$, solve for each such choice the system

$$
\left[\begin{array}{cccc}
x_{1} & y_{1} & z_{1} & -\lambda / U \\
x_{2} & y_{2} & z_{2} & 0 \\
x_{3} & y_{3} & z_{3} & 0 \\
x_{4} & y_{4} & z_{4} & 0
\end{array}\right]\left[\begin{array}{c}
x \\
y \\
z \\
m_{1}
\end{array}\right]=\left[\begin{array}{c}
\Phi_{1} / U \\
\left(\Phi_{2}+m_{2} \lambda\right) / U \\
\left(\Phi_{3}+m_{3} \lambda\right) / U \\
\left(\Phi_{4}+m_{4} \lambda\right) / U
\end{array}\right]
$$

and check whether the solution satisfies the conditions $x^{2}+y^{2}+z^{2}=1$ and $m_{1} \in \mathbb{Z}$; however, this still means going through $12^{3}=1728$ possible cases. Thus the typically used vector-based approaches are seen to be very inefficient, even in this simplified example. In general, the computing time of our goniometric approach is negligible compared to the computing time of vector-based methods. We emphasize that the superiority of our solution to existing ones stems from the treatment of directions not as vectors, but as entities in their own right.

Acknowledgement. We are greatly indebted to Dieter Kilsch (Fachhochschule Bingen) and Norbert Wiengarn (European Space Operations Centre) for reading early drafts of this paper and for pointing out to us several relevant references, and to Julika Bertram (Fachhochschule Wiesbaden) for creating the drawings for the paper.

\section{References}

[1] Berger, M.: Geometry. Vol. II, Springer-Verlag, Heidelberg 1987.

[2] Cohen, C.E.: Attitude Determination Using GPS. PhD Thesis, Stanford University, December 1992.

[3] Cracknell, A.P.: Crystals and Their Structures. Pergamon Press, Oxford-London-Edinburgh-New YorkToronto-Sydney-Paris-Braunschweig 1969.

[4] Darboux, G.: Principes de Géométrie Analytique. Gauthier-Villars, Paris 1917.

[5] Dittmer, A.: Cross Product Identities in Arbitrary Dimension. Amer. Math. Monthly (1994), 887-891.

[6] Fraiture, L.: Spherical Trigonometry for GPS Ambiguity Resolution. Proc. 10th Brazilian Colloquium on Orbit Dynamics, Nazare Paulista, Nov. 20-24, 2000.

[7] Gallot, S.; Hulin, D.; Lafontaine, J.: Riemannian Geometry. Springer-Verlag, Berlin-Heidelberg-New York 1987.

[8] Hazewinkel, M. (ed.): Encyclopaedia of Mathematics. Kluwer Academic Publishers, Dordrecht-BostonLondon 1989.

[9] Horn, R.A.; Johnson, C.R.: Matrix Analysis. Cambridge University Press, Cambridge-London-New York-New Rochelle-Melbourne-Sydney 1985.

[10] Horn, R.A.; Olkin, I.: When Does $A^{\star} A=B^{\star} B$ and Why Does One Want to Know? Amer. Math. Monthly, Vol. 103 (1996), 470-482.

[11] Jacobson, N.: Lectures in Abstract Algebra. Vol. III: Theory of Fields and Galois Theory. Van Nostrand/Reinhold, New York-Toronto-London-Melbourne 1964.

[12] Knight, D.: A New Method of Instantaneous Ambiguity Resolution. ION GPS-94, 707-715, Salt Lake City, 1994.

[13] Menardi, A.S. et al.: Comprehensive Report on the Horizon Crossing Indicator Flown on ESRO IV. ESA TR-15, Paris 1977.

[14] Norkin, C.C.; White, D.J.: Measurement of Joint Motion: A Guide to Goniometry. Davis, Philadelphia 1985.

[15] Phillips, F.C.: An Introduction to Crystallography. 3rd ed., John Wiley \& Sons, New York 1963. 
[16] Shuster, M.D.: Attitude Determination in Higher Dimensions. J. of Guidance, Control, and Dynamics 16(2) (1993), 393-395.

[17] Wertz, J.R. (ed.): Spacecraft Attitude Determination and Control. Kluwer Academic Publishers, Dordrecht 1978.

Luc Fraiture

Flight Control Systems Department

European Space Operations Centre

D-64293 Darmstadt, Germany

e-mail: lucfraiture@web.de

Karlheinz Spindler

Fachbereich MNDU

Fachhochschule Wiesbaden

D-65197 Wiesbaden, Germany

e-mail: spindler@r5.mnd.fh-wiesbaden.de 